\title{
Ontogeny of neural circuits underlying spatial memory in the rat
}

\author{
James A. Ainge ${ }^{1}$ and Rosamund F. Langston ${ }^{2 *}$ \\ School of Psychology, University of St. Andrews, St. Mary's Quad, St. Andrews, Fife, Scotland, UK \\ 2 Division of Neuroscience, Medical Research Institute, University of Dundee, Ninewells Hospital and Medical School, Dundee, Scotland, UK
}

\section{Edited by:}

Lisa M. Giocomo, Norwegian

University of Science and

Technology, Norway

Reviewed by:

Paul A. Dudchenko, University of Stirling, UK

Giorgio Ascoli, George Mason

University, USA

\section{*Correspondence:}

Rosamund F. Langston, Division of Neuroscience, Medical Research Institute, University of Dundee, Ninewells Hospital \& Medical School, Dundee DD1 9SY,

Scotland, UK.

e-mail: r.f.langston@dundee.ac.uk
Spatial memory is a well-characterized psychological function in both humans and rodents. The combined computations of a network of systems including place cells in the hippocampus, grid cells in the medial entorhinal cortex and head direction cells found in numerous structures in the brain have been suggested to form the neural instantiation of the cognitive map as first described by Tolman in 1948. However, while our understanding of the neural mechanisms underlying spatial representations in adults is relatively sophisticated, we know substantially less about how this network develops in young animals. In this article we briefly review studies examining the developmental timescale that these systems follow. Electrophysiological recordings from very young rats show that directional information is at adult levels at the outset of navigational experience. The systems supporting allocentric memory, however, take longer to mature. This is consistent with behavioral studies of young rats which show that spatial memory based on head direction develops very early but that allocentric spatial memory takes longer to mature. We go on to report new data demonstrating that memory for associations between objects and their spatial locations is slower to develop than memory for objects alone. This is again consistent with previous reports suggesting that adult like spatial representations have a protracted development in rats and also suggests that the systems involved in processing non-spatial stimuli come online earlier.

Keywords: hippocampus, memory and learning, postnatal development, entorhinal cortex, spatial representation, place cell, grid cell, head direction cell

\section{INTRODUCTION}

The ability of animals to internally represent external space and use this representation to guide navigation-based behavior was first suggested by Tolman in his proposal of the cognitive map theory in 1948 (Tolman, 1948; O'Keefe and Nadel, 1978). Since then a number of systems within the hippocampus and surrounding regions of the medial temporal lobe have been discovered which provide the basic building blocks that could support such a system. Place cells in the hippocampus (O'Keefe and Dostrovsky, 1971) combined with grid cells (Hafting et al., 2005), head direction cells (Taube et al., 1990; Sargolini et al., 2006) and border cells (Savelli et al., 2008; Solstad et al., 2008), which have been found in other areas of the medial temporal lobe, provide all the information that an animal would need to navigate efficiently within familiar environments. Some aspects of these systems have been extensively studied. A large amount of research has been carried out to examine the properties of place cells including how they respond to different familiar environments (Muller, 1996; Lever et al., 2002; Leutgeb et al., 2005a,b; Wills et al., 2005; Colgin et al., 2008), changes in physical characteristics of environments (Muller and Kubie, 1987; Bostock et al., 1991; O'Keefe and Burgess, 1996) and changes in cognitive demands within an environment (Wood et al., 2000; Ferbinteanu and Shapiro, 2003; Lee et al., 2006; Smith and Mizumori, 2006; Ainge et al., 2007a,b, 2012; Griffin et al., 2007). These studies have significantly enhanced our understanding of how place cells contribute to our ability to represent familiar spatial locations. Head direction cells have also been widely studied and their properties are well documented (for review see Taube, 2007). Grid cells and border cells have been discovered more recently and as such are much less well understood, although this situation is changing rapidly. However, a number of factors, such as the interdependence of these systems, remain to be addressed. Here we discuss a novel approach to this question, namely examining the ontogeny of the neural circuits for spatial representations. We present new data suggesting that the systems that support navigation and spatial memory come on line at similar points on the developmental timescale.

\section{ONTOGENY OF SPATIAL MEMORY}

Following the discovery of place cells, much research was directed toward spatial memory and the mechanisms underlying it in adult rodents. However, relatively little research has examined how this type of memory develops in young animals. Those studies that have examined this development have used the same behavioral paradigms as used in adults, primarily the Morris water maze.

One of the most comprehensive studies of spatial memory in young rats was carried out by (Schenk, 1985) in which rats of different ages were trained to navigate to a hidden platform in the Morris water maze using either distal environmental cues 
(place learning) or a proximal cue marking the location of the escape platform (cue and place learning). Rats began training at Postnatal day 21 (P21), P28, P35, P42, or as adults (P64) and were tested for 5 days. All age groups learned to escape from the pool more quickly when trained with the additional proximal cue compared to just using distal room cues, although all age groups learned the location of the escape platform in both the cue and place conditions. What was noticeable about the younger animals (P21 and P28 groups) was that if they were trained in the place only condition, they benefitted more from the later addition of a proximal cue than did older animals. Conversely, if they were trained with the additional proximal cue and this was later removed, their ability to find the hidden escape platform was impaired much more than that of older animals. These data imply that during the fourth and fifth postnatal weeks, rats are reliant on the presence of local visual cues in the environment to perform optimally, but after postnatal week 6 , they are much less affected by the manipulation of the local environment and are capable of using solely distal environmental cues to navigate effectively.

Akers et al. (2007) examined the development of navigationbased on directional headings. In their study, P24 rats were trained using a place strategy to find a hidden platform in the Morris water maze and then their memory for this location was tested in a series of probe tests. During the probe tests the physical location of the water maze was changed in relation to the distal cues in the environment (which the rats had used to learn the task). This manipulation aimed to discover whether the rats would use the distal cues in the room to navigate to the trained platform position as an absolute location in space or whether they would use the directional heading information they had learned from their starting point in the previous pool location and swim to the same platform location relative to the pool walls and start point. The authors found that the weanling rats used directional heading to navigate to the platform location, ignoring the distal room cues.

Other studies have gone on to examine further the extent to which even younger rats can navigate using cued locations or adult like allocentric memory. These studies demonstrated that rats as young as P17 were able to learn to navigate to an escape platform marked by a proximal cue (equivalent to the "cue and place" condition of Schenk, 1985) but that rats at this age showed no learning in a place only condition (Rudy et al., 1987). Rats aged P21 however already showed significant learning of the location of the escape platform in the place only condition. This experiment did not use adult control animals for comparison so does not indicate when cue and place learning reach adult levels but does show a differential ontogeny of the two types of learning, with place learning developing later than cue learning. These findings were disputed by Brown and Whishaw (2000) whose findings indicated that both place and cue learning can be displayed in rats as early as P19 (Brown and Whishaw, 2000). They do, however, concede that their measures of place learning revealed lower levels of performance relative to cued learning, therefore supporting Schenk's (1985) data that younger animals are more reliant on proximal visual cues than distal environmental cues.

\section{ONTOGENY OF SPATIAL REPRESENTATIONS}

Clearly rats' ability to learn and remember spatial locations is not present from birth but rather develops through adolescence into early adulthood. If place cells, grid cells and the other cell types in the medial temporal lobe are the critical mechanisms that underlie rats' ability to navigate and remember spatial locations then these should develop on a similar timescale to their spatial memory. In a recent study we addressed this issue by examining the timescale upon which place cells, grid cells, and head direction cells develop in very young rats (Langston et al., 2010). Rats typically start to explore their surroundings after they have opened their eyes on approximately P15. After this they start to make longer and more frequent journeys away from the nest to explore their external environment. This is consistent with the behavioral data which suggests that at the beginning of this exploration process (P17) rats have poor spatial memory but that this improves in the next 2-3 postnatal weeks. We used in vivo single unit electrophysiology to record place cells in the CA1 region of hippocampus, grid cells from MEC and head direction cells from presubiculum. By examining the timescale upon which these systems develop and comparing the spatial information that they convey with that conveyed by the adult system in the same environments we were able to see whether these systems develop on a timescale that is consistent with them being the critical mechanism underlying spatial memory. We were also able to address the interesting philosophical question of whether an understanding of space is an innate psychological capability or whether it develops with experience of the world. Finally we were able to ask questions about the hierarchy and interdependence of the place cell, grid cell, and head direction cell systems.

The results from our study are illustrated in Figure 1. Head direction cells were present in presubiculum in the very earliest recordings that were made (Figure 1A). These animals were 15 days old and had usually opened their eyes during the previous $24 \mathrm{~h}$. The particularly noteworthy properties of these head direction cells were that they conveyed as much directional information as head direction cells recorded from adult rats in the same environment and that the preferred direction of firing was consistent across sessions. To examine consistency we examined head direction information in two 10 min sessions. The rat was removed from the box between sessions and so by correlating the head direction information between the sessions we were able to examine how consistently individual head direction cells represented a directional heading within a familiar environment. The results showed that rats have an adult like ability to remember a directional heading as soon as they open their eyes. While it remains a possibility that only a very small amount of visual experience was necessary to form this directional representation these data suggest that this psychological capability may be innate.

We then went on to look at place cells during the same developmental period. Place cells had some adult properties in the very young rats but other properties took longer to develop. As shown in Figure 1, place cells that had specific spatial firing patterns were present at the earliest time point that we were able to sample (P17; Figure 1B). These cells conveyed similar amounts of spatial information to place cells in adult rats in the same environment. 
A
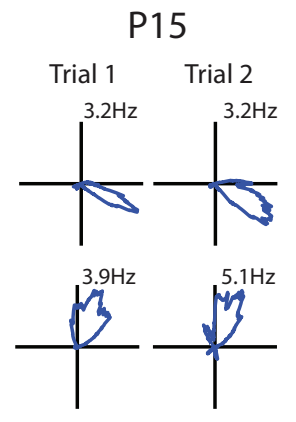

C
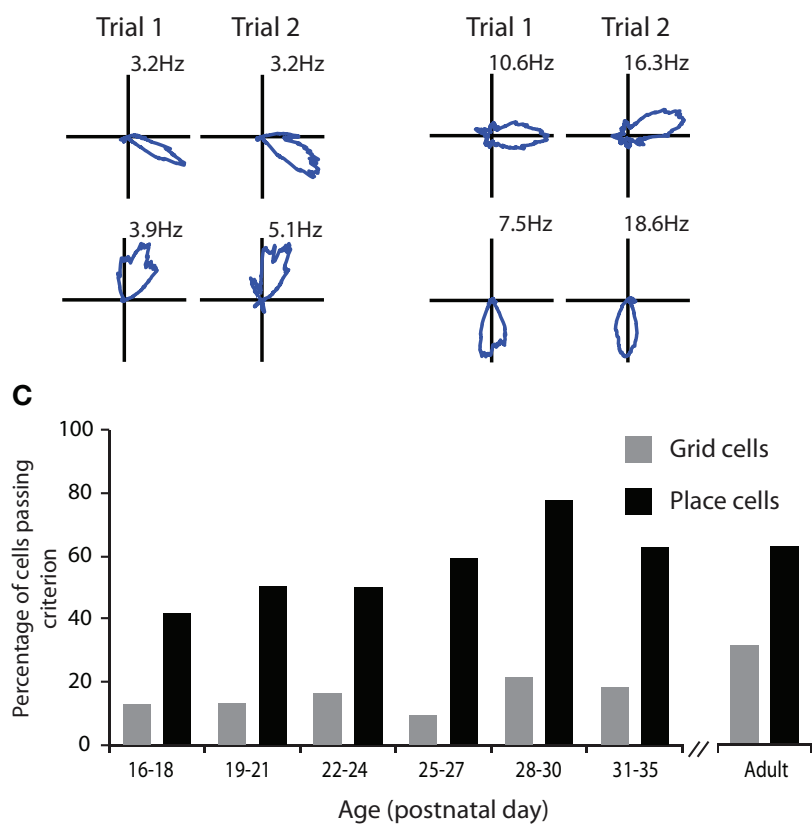

B

Grid cells
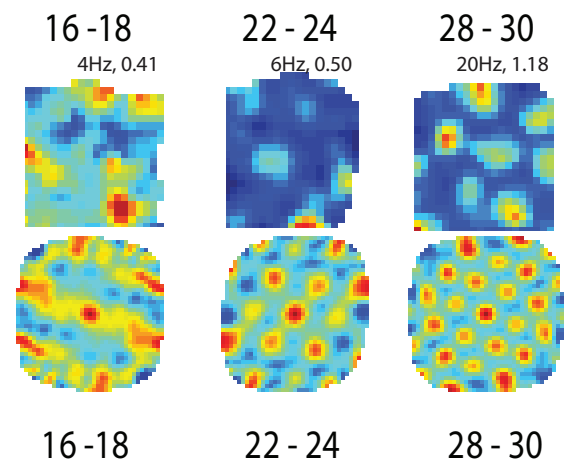

Place cells

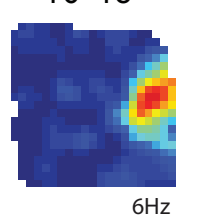

$28-30$

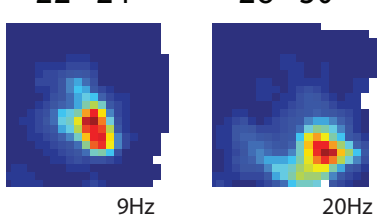

FIGURE 1 | Spatial representations at the outset of navigational experience. (A) Head direction cells show adult like directional coding and stability at P15. (B) Grid cells show grid like characteristics in their rate maps (top row) and spatial autocorrelations (middle row) from P16 to 18 but the regularity and specificity of the grid increases with age.
Examples of adult like place cells from P16 to 18 (bottom row). (C) Percentage of cells passing criterion for being place and grid cells through development. Note that the proportion of both place and grid cells that pass the criterion increases with age. Adapted from Langston et al. (2010).
However, the proportion of cells that conveyed enough spatial information to satisfy our criterion for being place cells increased through the first 4-5 post natal weeks (Figure 1C). This suggests that the network takes some time to develop an adult like representation of space. When the consistency of place cell firing across sessions was examined it was again clear that while very young rats had some place cells that showed consistent firing location across trials the proportion of these cells increased to adult levels through the first 4-5 postnatal weeks. This finding is consistent with the studies showing that rats' use of allocentric cues from the environment to guide navigation is immature until at least the third or fourth postnatal week (Schenk, 1985; Rudy et al., 1987).

Finally we examined grid cell firing through the course of development. As with the other two cell types we examined it was clear from the earliest recordings that some cells with grid like properties were present at this very early stage of development (Figure 1B). However, when we measured how regular grid cell firing was it was clear that grid cell regularity increased throughout the first 4-5 weeks of postnatal development (Figure 1B). This was combined with an increase in the number of cells that had regular enough firing to pass our criterion for being a grid cell. As with the place cell data, grid cell consistency across sessions also increased across the first 4-5 postnatal weeks. These data suggest that while some grid like cells are present from a very early age the adult like grid cell representation takes a long time to develop. This seems to give a clear hierarchy of cell types within the hippocampal-entorhinal system. Head direction cells develop first followed by place cells and finally grid cells. This hierarchy would be consistent with some computational models which suggest that grid cells are formed from inputs from head direction cells and that their stability is reliant on projections from the place cell system in the hippocampus and information about environmental boundaries (Burgess et al., 2007; Hasselmo et al., 2007). However, at first glance these data appear to be at odds with other models that suggest that place cell firing patterns are derived from the spatial information in the grid cells (Solstad et al., 2006; Monaco and Abbott, 2011). Given that grid cells develop adult like properties relatively slowly it seems unlikely that they are the source of the spatial information needed to form adult like place cells. However, this interpretation may be somewhat simplistic. It is entirely conceivable that place cells could be formed from fairly coarse spatial information from grid cell like firing patterns such as those seen in the very early recordings. It is also the case that lesions of the hippocampus do not abolish grid cell firing in the MEC which suggests that grid cells are not reliant on place cell input (Fyhn et al., 2004) or can at least compensate for the absence of the majority of place cell input. Inactivation of place cells does, however, disrupt grid cell firing and in some cases cells that used to have grid like properties become head direction cells (Bonnevie et al., 2006). This would be consistent with the hypothesis that grid cells are formed by combining inputs from head direction cells and that the grid representation is stabilized by place cell input. 
The fine-tuning of the spatial signal in the medial entorhinal cortex suggests that some properties of the system mature during the time period when the rat starts to explore its external surroundings. To attempt to identify these properties we went on to record from groups of 3-4 unconnected stellate cells in slices from littermates of the rats in the in vivo recording study at the same age. Spontaneous changes in the subthreshold membrane potentials were then examined and correlated across pairs of simultaneously recorded cells. At the early time points between P16-P21 there were relatively few pairs of cells with significantly correlated subthreshold changes in membrane potential. However, the proportion of cell pairs with significantly correlated subthreshold membrane potential changes increased to between $30 \%$ and $40 \%$ by P29. In contrast the strong inputs to MEC from presubiculum have adult like properties from 2 weeks of age. This suggests that the maturation of the grid cell signal in MEC is dependent on the intrinsic connectivity of MEC rather than just reflecting a maturation of its afferent connections.

Our data are largely consistent with other reports of spatial representations throughout the postnatal developmental period. Wills et al. (2010) reported similar findings in that directional firing properties were present at very early ages while place cells and grid cells develop more slowly in the third and fourth postnatal week. As in our study Wills et al. showed robust theta activity in the local field potential from the very earliest recordings although the frequency of the theta did increase significantly with age independently of running speed. Two other studies have examined place cells during development (Martin and Berthoz, 2002; Scott et al., 2011). These data are somewhat contrasting with our own and that of Wills et al. in that they suggest that place cells carry on developing for much longer. Indeed it was suggested that place cells do not fully mature until approximately $\mathrm{P} 40-\mathrm{P} 45$. The findings of Martin and Berthoz are based on very few cells and as such are hard to interpret as the variability in the sample is large. Scott et al., however, recorded from a large population of place cells across a range of ages. There is some agreement across studies in that all of the available data suggest that some properties of place cells (stability in particular) are relatively slow to mature. However, the data from Wills et al. combined with our own clearly demonstrate that place cells that provide adult like levels of spatial information are present from as young as P17 and indeed the spatial information content of the cells that pass criterion for being place cells does not increase with age.

\section{DIFFERENTIAL ONTOGENY OF OBJECT AND ASSOCIATIVE SPATIAL MEMORY IN THE JUVENILE RAT}

These electrophysiology data have demonstrated that rudiments of the network of systems that support representations of external space are present in young rats at the outset of navigational experience. While some aspects of these systems have adult like qualities from the day that the rats open their eyes, other aspects take longer to mature and do not reach adult levels of specificity and stability until the fourth or fifth postnatal week. These data are consistent with studies showing that rats can navigate using direction information from very young ages but the ability to use allocentric spatial memory does not develop until after some experience of exploring the world. These studies have concentrated on spatial memory and navigation but the same neural systems have been suggested to have a critical role to play in more general memory processes, specifically episodic memory, in both humans and rats (Vargha-Khadem et al., 1997; Eichenbaum et al., 1999; Gelbard-Sagiv et al., 2008; Langston and Wood, 2010). It has been suggested that the role of the hippocampus is to bind features of events we have experienced, including spatial location, together to form episodic memories (Eichenbaum et al., 2011). We have recently addressed this issue using the developing rat as a model to examine whether memory for single features of an event (objects) is evident earlier in development than associative memory for multiple features of an event (object in location). The evidence reviewed so far suggests that rats' ability to represent space takes a while to reach adult levels of maturity and consequently we would predict that memory for objects within specific spatial locations will be equally slow to develop. Memory for object identity has been demonstrated to be dependent on the perirhinal cortex (Brown and Aggleton, 2001). As this memory is not dependent on the hippocampus or entorhinal cortex it remains a possibility that this type of memory will not show the same slow emergence during development.

Memory for specific features of an environment can be tested using the object recognition paradigm in which rats are presented with novel junk objects and given the opportunity to explore them. Rats have been shown to have a propensity to preferentially explore novel objects relative to familiar objects within a familiar environment (Ennaceur and Delacour, 1988). This task has been adapted in a number of ways to examine different types of memory and studies have shown that rodents will preferentially explore familiar objects within novel locations in preference to the same objects presented in familiar locations. This demonstrates a memory for the combination of object and spatial location in which it was presented (Dix and Aggleton, 1999). To examine memory for objects within spatial locations through development we used tests of object recognition and object in place recognition at different postnatal ages.

\section{MATERIALS AND METHODS SUBJECTS}

Twelve Lister Hooded rats were used as subjects. Six rats were tested on postnatal day (P) 24 and six rats were tested on P30. After being weaned from their mother at P21 they were housed in single sex groups of 3-6, and kept on a $12 \mathrm{~h}$ light/12 h dark cycle ("sunrise" 5-6 a.m., "sunset" 5-6 p.m.). All rats had unrestricted access to food and water throughout the experiment. Compliance was ensured with national (Animals [Scientific Procedures] Act, 1986) and international (European Communities Council Directive of 24 November 1986 [86/609/EEC]) legislation governing the maintenance of laboratory animals and their use in scientific experiments.

\section{APPARATUS}

All testing was carried out in a square arena measuring $70 \times$ $70 \mathrm{~cm}$ with walls $40 \mathrm{~cm}$ in height. The arena was constructed of wood and painted with brown matt floor paint (Johnstone's, UK). 
Two $3 \mathrm{~cm}$ strips of Dual-Lock (3M, UK) reusable adhesive were attached to the floor in the two locations in which objects were to be presented in the arena, in order to secure the objects to the arena floor and prevent the rats from displacing them. These locations were $10 \mathrm{~cm}$ from the box walls, at the north-west and north-east points. The arena was placed on a table approximately $50 \mathrm{~cm}$ high to allow ease of access for the experimenter. The arena was open to the room to allow the rats access to distal visual room cues. There were also two proximal cues situated above the northwest and north-east corners of the arena. These were a plastic plant pot approximately $10 \mathrm{~cm}$ diameter and $15 \mathrm{~cm}$ high and a plastic model of a tree approximately $15 \mathrm{~cm}$ high. These proximal cues were suspended from the ceiling of the testing room using string so that they hung with their bases just inside the top of the walls of the arena, with the aim that the rats could use them as visual cues but not physically reach them and interact with them. There was a holding container approximately $40 \mathrm{~cm}$ high with a floor area of $15 \times 25 \mathrm{~cm}$ covered by a $2 \mathrm{~cm}$ layer of woodchip bedding (as used in the rats' home cages) which was used to house the rats in between the different stages of testing (described in the next section) placed in a corner of the room approximately 2 meters away from the testing arena. The room was lit by two fluorescent strip lights on the ceiling, approximately equidistant from the testing arena. There was a radio in the testing room which was kept on at a constant location, frequency and volume during testing with the aim of providing low-level background noise in the room to minimize the possibility of the rats becoming startled by noises in adjacent rooms and corridors.

Objects for exploration were collected from a variety of sources but had to fulfill the criteria of being easily cleaned, made from non-porous materials and having a suitable flat base where a reusable adhesive strip could be attached. Object size and shape varied but followed the guidelines that objects should either be larger than the rat in one or more dimensions or have complex surfaces/features. These guidelines were formed from previous experience and observations (unpublished) of rats interacting with three-dimensional objects.

\section{BEHAVIORAL TESTING}

We shortened a previously published protocol for novel object recognition and associative spatial recognition memory testing which is normally carried out over 2 weeks in adult rats (Langston and Wood, 2010) into a 2 day protocol in order to be able to test specific time points during development in juvenile rats. All behavioral testing was carried out in the light phase between 8 a.m. and 5 p.m.

\section{Handling}

Each rat was handled for three daily sessions prior to commencing the experiment. The aim of this stage was to familiarize the rats with the experimenter and the procedure of being removed from and replaced in the home cage. The handling consisted of the experimenter picking up, holding, manipulating and replacing each rat multiple times each day with the total contact time for each rat being around 2 min per day. For the older group of rats (P30), handling was carried out on P26, P27, and P28. For the younger group of rats (P24), handling was carried out on P20 (while the rats were still housed with their parents), P21 (weaning day) and P22.

\section{Habituation}

One group habituation session and one individual habituation session were carried out on the day after the last handling session, when the rats were aged P29 (older group) and P23 (younger group). There were no objects in the arena during the habituation sessions.

The group habituation session was carried out in the morning and consisted of all rats who were housed in a cage together being placed into the testing arena together for $15 \mathrm{~min}$. The arena was cleaned between cage groups using lemon scented cleaning wipes (Tesco, UK) to minimize distracting odors from the previous occupants of the arena and provide a consistent olfactory environment within the arena. The aim of this stage was to begin to familiarize the rats with the testing arena and to reduce stress by allowing them to remain with their cagemates. The individual habituation session was carried out in the afternoon of the same day. Each rat was individually placed into the testing arena for $5 \mathrm{~min}$. The arena was cleaned between each rat using lemon scented cleaning wipes. The aim of this stage was to further familiarize the rats with the testing arena and give them the experience of being in the arena alone.

On both habituation sessions, the rats entered the testing arena from the south, and were placed in to the arena facing the south wall.

\section{Novel object recognition}

The day after habituation (P30 and P24 for the older and younger groups respectively), the rats were tested on a standard novel object recognition task during the morning (Figure 2A, upper schematics). Each rat was removed from its home cage and placed in the holding container while the apparatus was configured. Two copies of the same object were cleaned with lemon scented cleaning wipes and attached at the north-west and north-east locations in the testing arena (Figure 2A, top left schematic). The rat was placed into the arena from the south side, facing the south wall, for the sample phase. The sample phase consisted of the rat being allowed to explore the two identical objects in the testing arena for $2 \mathrm{~min}$ Exploration was defined as the rat being within $2 \mathrm{~cm}$ of an object, directing its nose at the object and being involved in active exploration such as sniffing or whisking. Sitting on or next to an object without any signs of active exploration was not included. After 2 min the rat was removed from the arena at the same point from which it entered and placed in the holding container for a 1-2 min intertrial interval while the arena was reconfigured for the test phase. The floor and walls were cleaned and new objects were cleaned and placed in the arena. For the test phase one object was a third copy of the two objects seen in the sample phase while the other object was completely novel (Figure 2A, top right schematic, novel object marked by an arrow). The test phase was carried out using exactly the same procedures as the sample phase. After the test phase, the rat was returned to its home cage. The location and identity of the novel object was counterbalanced across each age group of rats. 
A

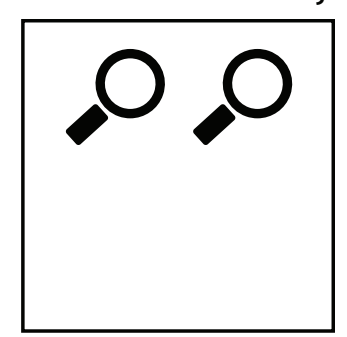

Object recognition

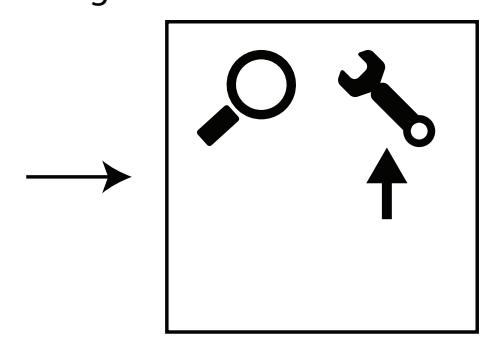

Associative spatial recognition (object-location)
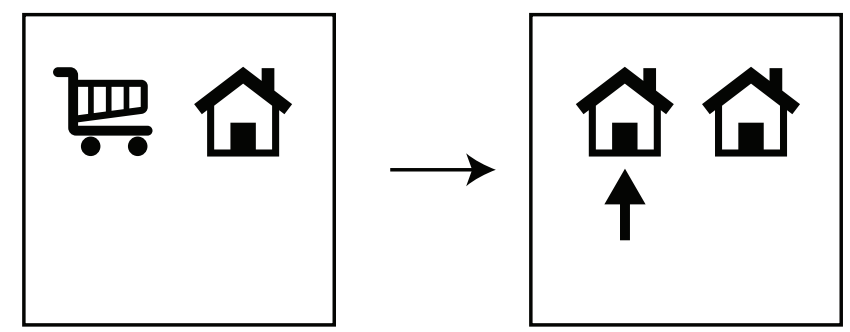

C

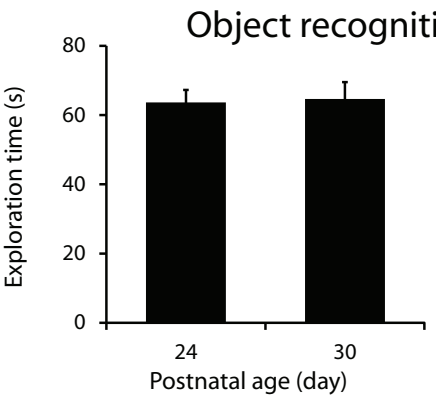

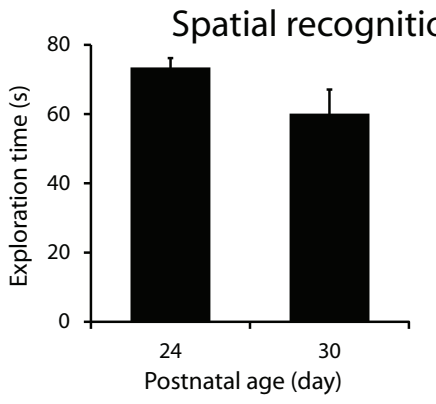

B

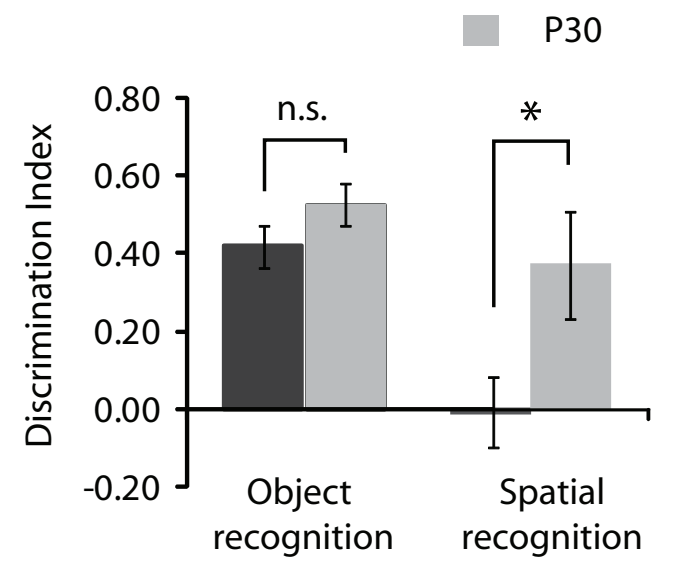

FIGURE 2 | Development of memory for objects and objects in location. (A) Object recognition protocol (top row) and associative spatial recognition protocol (bottom row). Both tasks consist of a sample phase where rats are presented with two objects to explore (left schematics) followed by a 2 min delay and a test phase (right schematics) where rats are given different combinations of objects to test memory for object or object in location. Arrows in test phase schematics represent the novel object or combination of object and location that we would expect the rats to

\section{Associative spatial recognition (object-location task)}

In the afternoon of the same day as the novel object recognition task the rats were tested on an associative spatial recognition task. The procedure for the rats in this task was the same as that used in the novel object recognition task, except that the objects used in the sample and test phases were manipulated in the opposite way (Figure 2A lower schematics). During the sample phase, two different objects were present in the testing arena (Figure 2A, bottom left schematic). During the test phase, a further two copies of one of the objects from the sample phase were present. Thus, during the test phase, one of the objects was presented in the same location as it had been in the sample phase, whereas the other was in a location which had previously been occupied by a different object (Figure 2A, bottom right schematic, novel objectlocation configuration marked by an arrow). In this situation, both the objects and the two locations had been experienced during the sample phase, and so were familiar, but for one object, preferentially explore. (B) Performance on the object recognition and associative spatial recognition tasks. P30 rats showed significantly greater preference for the novel object-location association than the P24 rats ( $p<0.05$ ) while the groups did not differ in standard object recognition performance. (C) Total exploration of the objects in the sample phase of the two tasks. Note no significant difference between the groups and the trend is toward P24 rats showing greater levels of exploration. the object-location configuration was the same as in the sample phase, but for other it was novel. The location and identity of the novel configuration was counterbalanced across each age group of rats.

\section{DATA COLLECTION}

An overhead black and white camera was used to monitor the movement of the rat around the testing arena. The video signal was fed into a TV monitor on the desk of the experimenter. A computer ran an in-house timing program whereby depression of a key on the computer keyboard would activate a timer. This was performed manually by the experimenter who observed the behavior of the rat via the TV monitor and recorded the amount of time the rat was engaged in exploration. Key presses activated timers which differentially timed exploration at the left and right objects. The raw data from sample and test phases were recorded as times that rats spent exploring the left and right objects. Video 
files of all data were recorded on to a hard drive connected to the TV monitor so that a random selection of the tests could be rescored and checked for accuracy by an experimentally blind second analyst.

\section{DATA ANALYSIS}

Data from the novel object recognition and associative spatial recognition tasks were analyzed in the same way. The raw exploration times at each object were converted into a discrimination index for each rat on each task using the formula (time at novel time at familiar)/(time at novel + time at familiar) where novel refers to the novel object, or the novel configuration of object in place in the associative spatial recognition task, and familiar refers to the other object. A value of zero indicates no preference, whereas a positive value indicates preferential exploration of the novel configuration and a negative value indicates preferential exploration of the familiar configuration.

Statistical analyses of the discrimination index values were run in SPSS. A repeated measures ANOVA was performed with age group (P24 vs. P30) as the between subjects factor, task (object vs. spatial recognition) as the within subjects factor and discrimination index as the dependent variable. Post hoc tests (independent samples $t$-tests) were carried out to examine whether rats of different ages differed in their preference for the novel object (object recognition) or novel configuration of object and location (associative spatial recognition). Performance of each group was compared to chance level performance using 1-sample $t$-tests. Data collected during the sample phases of each task was also analyzed to examine the total amount of time spent exploring objects. Repeated measures ANOVA were performed for the sample and test phases of the tasks, with age group (P24 vs. P30) as the between subjects factor, task (object vs. spatial recognition) as the within subjects factor and total exploration time as the dependent variable.

\section{RESULTS}

The main result is illustrated in Figure 2B. We found that at P24, rats could successfully perform the novel object recognition task but performed at chance levels on the associative spatial recognition task. By P30 however, rats could successfully perform both memory tasks. Repeated measures ANOVA showed significant main effects of both group [P24 vs. P30; $F(1,10)=10.84$, $p<0.008$ ] and task [object recognition vs. associative spatial memory; $F(1,10)=8.79, p<0.014]$ and a significant group $\times$ task interaction $[F(1,10)=6.24, p<0.032]$. Post hoc 1 -sample $t$-tests revealed that rats of both ages spent significantly longer exploring the novel object than would be expected by chance $(p<0.05)$ in the object recognition task and that preference for the novel object did not differ between P24 and P30. In the associative spatial recognition task P30 rats showed a significantly greater preference for the novel configuration of object in location than the $\mathrm{P} 24$ rats $[t(10)=-3.263, p<0.009]$. P30 rats spent significantly longer exploring the novel configuration of object and location than would be expected by chance while the P24 rats' preference for the novel configuration did not differ from chance.

In order to check whether differences in memory performance (discrimination index) between P24 and P30 could be explained by variation in baseline exploratory levels, the total amounts of exploration in both the sample and test phases of each task were analyzed using repeated measures ANOVA. Analysis of both sample and test phase data indicated no significant main effect of task or group and no task $\times$ group interaction (Figure $2 \mathrm{C}$ ).

\section{DISCUSSION}

In summary, rats of both ages showed a preference for exploring the novel object in the standard test of object recognition. Clearly even young rats are capable of remembering objects that they have previously encountered over short periods of time. Rats at P30 also showed a preference for the copy of the object in the novel location in the associative spatial memory test. These rats showed a clear memory for the association between object and spatial location that they saw in the sample phase. However, P24 rats showed no preference for either object in the associative spatial memory test suggesting that they have no memory for where they have previously encountered familiar objects. These data fit with previous data in that they suggest that P24 rats do not have a fully functioning representation of external space into which event information, such as objects encountered, can be incorporated. By $\mathrm{P} 30$, however, rats can remember where familiar objects have been previously encountered suggesting that they do have a spatial framework that allows them to remember where they were when they encountered specific stimuli. One potential caveat is that young rats might show generally lower levels of interaction with the objects due to fatigue, anxiety, or lack of motivation. However, as illustrated in Figure 2C the P24 rats actually showed more total exploration of the objects than the P30 rats, although this difference was not significant. This argues against the lack of novelty preference being due to non-mnemonic factors such as fatigue or lack of motivation.

Interestingly these data are not consistent with a previous study of this type of memory in adolescent mice. Ricceri et al. (2000) tested mice at $18,28,46$, and 90 days old to see whether they could remember familiar objects in unfamiliar locations. Surprisingly it was reported that mice could not remember this form of associative spatial memory until they were 90 days old. These data are not consistent either with the current data or the studies examining navigation and spatial memory reviewed in the introduction This suggests that either mice develop spatial memory much later than rats or that the exact protocol used in this study is not sensitive enough to detect spatial memory at younger ages. Ricceri et al. presented mice with four objects at a time, some of which were replaced and some of which moved position between trials. This placed a much greater memory load on the system and as such the delay in associative spatial memory may have been due to task difficulty rather than a difference in the fundamental mechanisms supporting spatial memory across species. The data we have reviewed present a consistent picture that some ability to internally represent external space is present in rats even before they have any navigational experience of the world. This is consistent with behavioral data suggesting that rats can use simple navigational strategies to solve spatial memory tasks even at very young ages (P17). However, adult like representations of space throughout the hippocampal-entorhinal network do not develop until the fourth or fifth postnatal week. Again this is consistent 
with behavioral data showing that rats do not learn to solve allocentric spatial memory tasks based on distal visual cues until later in development (4 weeks postnatal). We have also presented data that suggests that rats' ability to bind features of events or episodes that they have experienced into a spatial framework also takes some time to develop. This memory is present in P30 rats but absent in rats aged $\mathrm{P} 24$.

The current data combined with the previous electrophysiology and behavior studies begin to elucidate the timescale upon which spatial representations develop. These types of studies are important for a number of reasons. Philosophers have debated for hundreds of years whether or not our ability to represent external space is innate or must be learned through experience. This longstanding debate can be captured by contrasting two philosophical schools of thought; rationalism and empiricism. Rationalists, such as Immanuel Kant, suggest that our understanding of concepts such as spatial representation go beyond that which can be gained from sensory experience. Kant argued that our sense of time and space are innate constraints of thought rather than the result of experience of the world (Kant, 1781). This view is at odds with empiricists, such as David Hume, who argue that all of our knowledge and concepts come through experience (Hume, 1777). While this oversimplified explanation does not do justice to the philosophical complexities of the arguments it does demonstrate that our ability to internally represent external space has been a controversial subject for many years. In recent years modern neuroscience techniques have allowed us to address these issues empirically for the first time.

While these extreme views are interesting from a philosophical perspective, one other possibility is that some aspects of our neural representation of space are present at birth but that these mature with experience to an adult level of complexity. Assuming that the system undergoes some kind of improvement or refinement during development, then understanding the timescale upon which this happens is of importance for many reasons. Creating age appropriate models for basic science is one of these. One of the key milestones in systems neuroscience research has been the discovery and examination of long-term potentiation (LTP) as a neural mechanism underlying learning and memory (Bliss and Lomo, 1973). LTP has been studied extensively in many neural systems and the detailed molecular mechanisms underlying it are now well understood (Martin et al., 2000; Malenka and Bear, 2004). However, one aspect of much of this research that is problematic is that the animals from which the slices are taken are very young, typically 2-3 weeks of age (McCutcheon and Marinelli, 2009). The reason for this is that recording from brain slices taken from older animals has proved to be technically challenging with stable electrophysiological signals difficult to isolate and maintain. Consequently much of our knowledge of the mechanism that is widely cited as the molecular basis of memory comes from the brains of very young animals and yet this is used to make predictions about the learning and memory capabilities of adult animals. Given the problems of recording from slices taken from adult animals, one way of reconciling this problem is to examine the behavioral and psychological capabilities of very young animals. If young animals are capable of adult like learning and memory then the study of the molecular mechanisms in slices taken from young animals is a valid experimental approach. In contrast if very young animals are incapable of learning new information in an adult like manner then this discrepancy in the literature must be addressed.

It is also interesting to characterize the time course of the development of spatial representations and the cognitive map in terms of developmental disorders. There are a number of clinical conditions involving abnormalities of learning and memory during childhood including autism, attention deficit hyperactivity disorder, schizophrenia, developmental amnesia, and Down syndrome. In creating animal models to study these disorders we need to take into consideration the ages of the human patients at onset of the disorders we wish to study and therefore the equivalent ages of our animal models. Many animal models of childhood or developmental diseases are modeled in adult animals. This approach may miss crucial information regarding the changes occurring during development which potentially contribute to these disorders and putative therapeutic targets may consequently go undiscovered.

\section{USING THE POWER OF YOUTH TO RESTORE THE WISDOM OF OLD AGE}

Another very interesting reason to characterize the ontogeny of spatial representations is that it may be possible to use development as a tool to investigate the cellular and network mechanisms underlying certain types of learning and memory. We propose that an ideal system in which to examine spatial memory, for example, is one in which you can study an individual organism in a longitudinal fashion at time points before and after which the organism has acquired the capability that you want to study. It would then be possible to define a critical time window in which a particular memory capability develops and then study the neural mechanisms during that window in order to define what properties of the network are changing. This approach allows researchers to characterize the critical rate limiting steps in development and correlate these neural mechanisms with learning and memory capabilities. The majority of research that examines critical mechanisms underlying psychological function involves producing lesions or temporary pharmacological inactivations in the brain to examine functional deficits. However, this approach is not ideal as the system is damaged and the level of compensation from other networks is hard to quantify. By using the developing rat as a model it is possible to examine these functions in a healthy brain and to use a longitudinal, within subjects design. The search for critical mechanisms underlying spatial memory is particularly important as it is a component of episodic memory, deficits in which are a key early symptom of Alzheimer's disease and other neurodegenerative disorders (Salmon and Bondi, 2009). Discovering the critical networks and mechanisms underlying formation of episodic memory would provide putative therapeutic targets for disorders in later life.

\section{ACKNOWLEDGMENTS}

This research was supported by a Tenovus small project grant to RFL. The authors would like to thank Ms. Frances R. Butterworth, a Carnegie Vacation Scholarship student in the laboratory of Rosamund F. Langston, for assistance with behavioral data collection. 


\section{REFERENCES}

Ainge, J. A., Tamosiunaite, M. Worgotter, F., and Dudchenko, P. A. (2012). Hippocampal place cells encode intended destination, and not a discriminative stimulus, in a conditional T-maze task. Hippocampus 22, 534-543.

Ainge, J. A., Tamosiunaite, M., Woergoetter, F., and Dudchenko, P. A. (2007a). Hippocampal CA1 place cells encode intended destination on a maze with multiple choice points. J. Neurosci. 27, 9769-9779.

Ainge, J. A., van der Meer, M. A., Langston, R. F., and Wood, E. R. (2007b). Exploring the role of context-dependent hippocampal activity in spatial alternation behavior. Hippocampus 17, 988-1002.

Akers, K. G., Candelaria, F. T., and Hamilton, D. A. (2007). Preweanling rats solve the Morris water task via directional navigation. Behav. Neurosci. 121, 1426-1430.

Bliss, T. V., and Lomo, T. (1973). Long-lasting potentiation of synaptic transmission in the dentate area of the anaesthetized rabbit following stimulation of the perforant path. J. Physiol. 232, 331-356.

Bonnevie, T., Fyhn, M., Hafting, T., Moser, M. B., and Moser, E. I. (2006). Misalignment of entorhinal grid fields after hippocampal inactivation. Society for Neuroscience Abstracts, 68.1.

Bostock, E., Muller, R. U., and Kubie, J. L. (1991). Experience-dependent modifications of hippocampal place cell firing. Hippocampus 1, 193-205.

Brown, M. W., and Aggleton, J. P. (2001). Recognition memory: what are the roles of the perirhinal cortex and hippocampus? Nat. Rev. Neurosci. 2, 51-61.

Brown, R. W., and Whishaw, I. Q. (2000). Similarities in the development of place and cue navigation by rats in a swimming pool. Dev. Psychobiol. 37, 238-245.

Burgess, N., Barry, C., and O'Keefe, J. (2007). An oscillatory interference model of grid cell firing. Hippocampus 17, 801-812.

Colgin, L. L., Moser, E. I., and Moser, M. B. (2008). Understanding memory through hippocampal remapping. Trends Neurosci. 31, 469-477.

Dix, S. L., and Aggleton, J. P. (1999). Extending the spontaneous preference test of recognition: evidence of object-location and object-context recognition. Behav. Brain Res. 99, 191-200.

Eichenbaum, H., Dudchenko, P. A., Wood, E. R., Shapiro, M. L., and
Tanila, H. (1999). The hippocampus, memory, and place cells: is it spatial memory or a memory space? Neuron 23, 209-226.

Eichenbaum, H., Sauvage, M., Fortin, N., Komorowski, R., and Lipton, P. (2011). Towards a functional organization of episodic memory in the medial temporal lobe. Neurosci. Biobehav. Rev. Jul 23 [Epub ahead of print].

Ennaceur, A., and Delacour, J. (1988). A new one-trial test for neurobiological studies of memory in rats. 1: behavioral data. Behav. Brain Res. 31, 47-59.

Ferbinteanu, J., and Shapiro, M. L. (2003). Prospective and retrospective memory coding in the hippocampus. Neuron 40, 1227-1239.

Fyhn, M., Molden, S., Witter, M. P., Moser, E. I., and Moser, M. B. (2004). Spatial representation in the entorhinal cortex. Science 305, 1258-1264.

Gelbard-Sagiv, H., Mukamel, R., Harel, M., Malach, R., and Fried, I. (2008). Internally generated reactivation of single neurons in human hippocampus during free recall. Science 322, 96-101.

Griffin, A. L., Eichenbaum, H., and Hasselmo, M. E. (2007). Spatial representations of hippocampal CA1 neurons are modulated by behavioral context in a hippocampus-dependent memory task. J. Neurosci. 27, 2416-2423.

Hafting, T., Fyhn, M., Molden, S. Moser, M. B., and Moser, E. I. (2005). Microstructure of a spatial map in the entorhinal cortex. Nature 436, 801-806.

Hasselmo, M. E., Giocomo, L. M., and Zilli, E. A. (2007). Grid cell firing may arise from interference of theta frequency membrane potential oscillations in single neurons. Hippocampus 17, 1252-1271.

Hume, D. (1777). Inquiries Concerning Human Understanding and Concerning the Principles of Morals. Oxford: Clarendon Press.

Kant, I. (1781). Kritik Der Reinen Vernunft. Riga, Latvia: Hartknoch.

Langston, R. F., Ainge, J. A., Couey, J. J., Canto, C. B., Bjerknes, T. L., Witter, M. P., Moser, E. I., and Moser, M. B. (2010). Development of the spatial representation system in the rat. Science 328, 1576-1580.

Langston, R. F., and Wood, E. R. (2010). Associative recognition and the hippocampus: differential effects of hippocampal lesions on object-place, object-context and object-place-context memory. Hippocampus 20, 1139-1153.
Lee, I., Griffin, A. L., Zilli, E. A. Eichenbaum, H., and Hasselmo, M. E. (2006). Gradual translocation of spatial correlates of neuronal firing in the hippocampus toward prospective reward locations. Neuron 51, 639-650.

Leutgeb, S., Leutgeb, J. K., Barnes, C. A., Moser, E. I., McNaughton, B. L., and Moser, M. B. (2005a). Independent codes for spatial and episodic memory in hippocampal neuronal ensembles. Science 309, 619-623.

Leutgeb, S., Leutgeb, J. K., Moser, M. B., and Moser, E. I. (2005b) Place cells, spatial maps and the population code for memory. Curr. Opin. Neurobiol. 15, 738-746.

Lever, C., Wills, T., Cacucci, F., Burgess, N., and O'Keefe, J. (2002). Longterm plasticity in hippocampal place-cell representation of environmental geometry. Nature 416, 90-94.

Malenka, R. C., and Bear, M. F. (2004). LTP and LTD: an embarrassment of riches. Neuron 44, 5-21.

Martin, P. D., and Berthoz, A. (2002). Development of spatial firing in the hippocampus of young rats Hippocampus 12, 465-480.

Martin, S. J., Grimwood, P. D., and Morris, R. G. (2000). Synaptic plasticity and memory: an evaluation of the hypothesis. Annu. Rev. Neurosci. 23, 649-711.

McCutcheon, J. E., and Marinelli, M. (2009). Age matters. Eur. J. Neurosci. $29,997-1014$

Monaco, J. D., and Abbott, L. F. (2011). Modular realignment of entorhinal grid cell activity as a basis for hippocampal remapping. J. Neurosci. 31, 9414-9425.

Muller, R. U. (1996). A quarter of century of place cells. Neuron 17, 979-990.

Muller, R. U., and Kubie, J. L. (1987). The effects of changes in the environment on the spatial firing of hippocampal complex-spike cells J. Neurosci. 7, 1951-1968.

O'Keefe, J., and Burgess, N. (1996). Geometric determinants of the place fields of hippocampal neurons. Nature 381, 425-428.

O'Keefe, J., and Dostrovsky, J. (1971). The hippocampus as a spatial map. Preliminary evidence from uni activity in the freely moving rat. Brain Res. 34, 171-175.

O'Keefe, J., and Nadel, L. (1978). The Hippocampus as a Cognitive Map. New York: Oxford University Press.

Ricceri, L., Colozza, C., and Calamandrei, G. (2000). Ontogeny of spatial discrimination in mice: a longitudinal analysis in the modified open-field with objects. Dev. Psychobiol. 37, 107-118.

Rudy, J. W., Stadler-Morris, S., and Albert, P. (1987). Ontogeny of spatial navigation behaviors in the rat: dissociation of "proximal"- and "distal"-cue-based behaviors. Behav. Neurosci. 101, 62-73.

Salmon, D. P., and Bondi, M. W. (2009). Neuropsychological assessment of dementia. Annu. Rev. Psychol. 60, 257-282.

Sargolini, F., Fyhn, M., Hafting, T., McNaughton, B. L., Witter, M. P., Moser, M. B., and Moser, E. I. (2006). Conjunctive representation of position, direction, and velocity in entorhinal cortex. Science 312, 758-762.

Savelli, F., Yoganarasimha, D., and Knierim, J. J. (2008). Influence of boundary removal on the spatial representations of the medial entorhinal cortex. Hippocampus 18, 1270-1282.

Schenk, F. (1985). Development of place navigation in rats from weaning to puberty. Behav. Neural Biol. $43,69-85$.

Scott, R. C., Richard, G. R., Holmes, G. L., and Lenck-Santini, P. P. (2011). Maturational dynamics of hippocampal place cells in immature rats. Hippocampus 21, 347-353.

Smith, D. M., and Mizumori, S. J. (2006). Hippocampal place cells, context, and episodic memory. Hippocampus 16, 716-729.

Solstad, T., Boccara, C. N., Kropff, E., Moser, M. B., and Moser, E. I. (2008). Representation of geometric borders in the entorhinal cortex. Science 322, 1865-1868.

Solstad, T., Moser, E. I., and Einevoll, G. T. (2006). From grid cells to place cells: a mathematical model. Hippocampus 16, 1026-1031.

Taube, J. S. (2007). The head direction signal: origins and sensory-motor integration. Annu. Rev. Neurosci. 30, 181-207.

Taube, J. S., Muller, R. U., and Ranck, J. B. (1990). Head-direction cells recorded from the postsubiculum in freely moving rats. I. Description and quantitative analysis. J. Neurosci. 10, 420-435.

Tolman, E. C. (1948). Cognitive maps in rats and men. Psychol. Rev. 55, 189-208.

Vargha-Khadem, F., Gadian, D. G., Watkins, K. E., Connelly, A., Van Paesschen, W., and Mishkin, M. (1997). Differential effects of early hippocampal pathology on episodic and semantic memory. Science 277 , $376-380$ 
Wills, T. J., Cacucci, F., Burgess, N., and O'Keefe, J. (2010). Development of the hippocampal cognitive map in preweanling rats. Science 328, 1487-1488.

Wills, T. J., Lever, C., Cacucci, F., Burgess, N., and O'Keefe, J. (2005). Attractor dynamics in the hippocampal representation of the local environment. Science 308, 873-876.
Wood, E. R., Dudchenko, P. A., Robitsek, R. J., and Eichenbaum, H. (2000). Hippocampal neurons encode information about different types of memory episodes occurring in the same location. Neuron 27, 623-633.

Conflict of Interest Statement: The authors declare that the research was conducted in the absence of any commercial or financial relationships that could be construed as a potential conflict of interest.

Received: 10 December 2011; accepted: 17 February 2012; published online: 01 March 2012.

Citation: Ainge JA and Langston RF (2012) Ontogeny of neural circuits underlying spatial memory in the rat Front. Neural Circuits 6:8. doi: 10.3389/ fncir.2012.00008

Copyright (c) 2012 Ainge and Langston. This is an open-access article distributed under the terms of the Creative Commons Attribution Non Commercial License, which permits non-commercial use, distribution, and reproduction in other forums, provided the original authors and source are credited. 